\title{
Mobiluncus curtisii subsp holmesii
}

National Cancer Institute

\section{Source}

National Cancer Institute. Mobiluncus curtisii subsp holmesii. NCI Thesaurus. Code C86897.

A subspecies of anaerobic, Gram positive, curved rod shaped bacterium assigned to the phylum Actinobacteria and the species Mobiluncus curtisii. This subspecies is motile by multiple subterminal flagella, esterase lipase positive, reduces nitrate and fermentation of glycogen in the presence of serum produces pyruvate. M. curtisii subsp. holmesii is found in the vaginal tract and is a causative agent of vag initis. 